\title{
COLOMBIA Y SUS COMPROMISOS CON LA PRIMERA INFANCIA
}

\author{
COLOMBIA AND ITS COMMITMENTS TO THE EARLY CHILDHOOD
}

\section{Por: Nicolás Simarra Torres* Camilo Madariaga Orozco**}

* NICOLÁS SIMARRA TORRES.

Licenciado en Ciencias Sociales y Económicas de la Universidad del Atlántico. Especialista en Docencia Universitaria, Universidad Santo Tomás. Magíster en Desarrollo Social Universidad del Norte. Catversiclad del Norte. Catedrático Universidad de Cartagena y Universida Tecnológica de Bolívar. Cartagena, Colombia. E-mail simarra65@hotmail.com. ${ }^{* *}$ CAMILO MADARIAGA OROZCO.

Psicólogo. Doctor en educación, Universidad de Humanismo Cristiano de Chile. Investigador adscrito al grupo de investigaciones en desarrollo humano (GIDHUM), Universidad del Norte. Coordinador de la maestría en desarrollo social. Barranquilla, Colombia. E-mail: cmadaria@uninorte.edu.co
Artículo recibido: Noviembre 28 de 2011. Artículo aprobado: Enero 05 de 2012

\section{RESUMEN}

Los niños y niñas entre cero y seis años constituyen un grupo clave en el presente y futuro de cualquier nación. En este artículo se abordan los argumentos por los cuales es importante invertir en este periodo de la vida del ser humano, destacando programas relevantes en este ámbito y la incidencia de organismos internacionales en el tema. También se aborda la trayectoria de Colombia en la atención a la primera infancia, discriminando varios escenarios, los primeros, los recientes y el momento actual, lo que aporta una visión sobre los avances pero también los grandes retos a que se enfrenta el país.

Palabras Clave: Primera infancia, política pública, niño, niña, Colombia.

\section{ABSTRACT}

Children between zero and six years are a key group in the present and future of any nation. For this reason, this article focuses on the arguments of why it is important to invest in this period of human life, highlighting that there are relevant programs in this area and that an impact of international organizations on this subject exists. It also addresses what the history of Colombia related to the early childhood care is. This way, this article examines various scenarios, the first ones, the most recent and the current ones, a good sample which provides us an overview of how much the country has progressed on this subject but also it shows which ones are the major challenges the country is facing.

Key Words: Early childhood, Public policy, children, Colombia.

\section{INTRODUCCIÓN}

En este artículo se hace un recorrido por los avances de Colombia en la atención a la primera infancia y la evolución de sus políticas. En primer lugar, aborda la importancia de la inversión en la población menor de seis años, resaltando los aportes más relevantes, y considerando las posturas de algunos organismos multilaterales como el Banco Interamericano de Desarrollo, BID; también se registran varios programas internacionales y latinoamericanos que evidencian los resultados favorables de esta inversión.
Artículo de reporte de caso según clasificación Colciencias. 


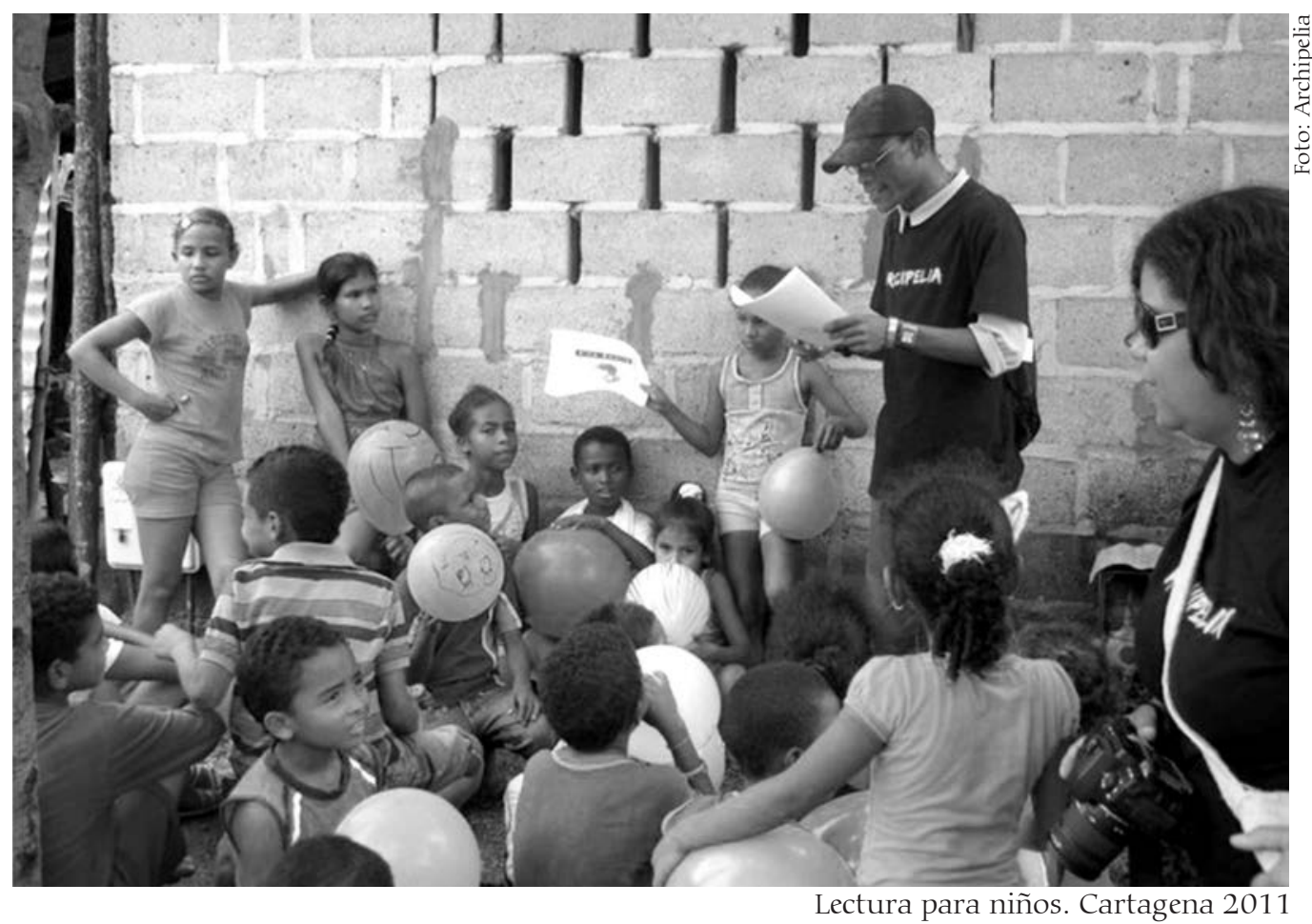

En segundo lugar, se hace una lectura de la apuesta que ha hecho Colombia por la primera infancia. Se revisan unos primeros escenarios donde se destaca la creación del Instituto Colombiano de Bienestar Familiar, ICBF, de programas como el Plan Nacional para la Supervivencia y el Desarrollo Infantil, SUPERVIVIR, y el Programa de Educación Familiar para el Desarrollo Infantil, PEFADI. Seguidamente se resalta el proceso de movilización a favor de la infancia en la primera década de este siglo y el diseño de la política Colombia por la Primera Infancia que se ratificó mediante el documento del Consejo Nacional de Política Social, CONPES 109 de 2007, que se recoge, en gran medida, en la Estrategia Nacional de Atención Integran a la Primera Infancia, De Cero a Siempre, impulsada por el actual gobierno.

Finalmente, se plantean unas conclusiones y recomendaciones que pueden ser útiles para avanzar en el análisis crítico de la atención integral a la primera infancia en Colombia.

\section{LA IMPORTANCIA DE INVERTIR EN PRIMERA INFANCIA}

La importancia de la inversión en los seis primeros años de vida ha sido objeto de análisis desde diferentes disciplinas y por numerosos expertos. Amartya K. Sen (1999) contribuyó a visibilizar el papel de esta inversión en el desarrollo de los pueblos, destacando la relación entre la inversión en primera infancia 
(en nutrición, inmunización y cuidado infantil, entre otros), y la reducción drástica de la mortalidad infantil.

Asimismo, es bien conocido el consenso en la literatura especializada sobre las implicaciones de las experiencias de los primeros años en el éxito que tendrán las personas durante la vida adulta. Los trabajos de Heckman (2010), Premio Nobel de Economía, constituyen grandes aportes al tema, demostrando que la inversión en los niños durante los primeros años produce efectos positivos para el país, "invertir en recursos educativos y que promuevan el desarrollo de familias en desventaja, para proveer el acceso equitativo al desarrollo humano en los primeros años de vida... asegura que tendremos ciudadanos más capaces, productivos y valiosos que traerán beneficios para nuestra sociedad y las generaciones futuras" (p. 4).

En la misma dirección, Mustard (2003) expuso que "las experiencias a las que se ve expuesto el recién nacido influyen directamente sobre el desarrollo cerebral, viéndose que las experiencias no estimulantes y pobres del medio ambiente durante la temprana infancia pueden llevar tanto a un coeficiente intelectual menor, a habilidades verbales y matemáticas pobres, a conductas antisociales, así como a problemas físicos y mentales en la vida adulta" (85), lo cual indica que el desarrollo exitoso del cerebro y de las habilidades y potenciales de los individuos depende del ambiente en el que el niño se desenvuelve.

El Banco Mundial (2002) posicionó el planteamiento que "la sociedad se beneficia del éxito alcanzado por los niños que logran un desarrollo adecuado y pueden incrementar su productividad, reduce el costo de tratar problemas psicosociales asociados a un desarrollo inadecuado como la delincuencia y otras conductas sociales perjudiciales como el uso de alcohol y drogas, y además reduce la posibilidad de que el niño se convierta en una carga social y de salud pública presupuestaria". (Prefacio p. V).

En relación con lo anterior, Mary Young (2003), especialista de ese organismo multilateral, señala que las oportunidades durante los primeros años sí hacen la diferencia. Asigna un lugar de privilegio a los programas de Desarrollo Infantil Temprano y a la necesidad de un esfuerzo de orden político sostenido para la expansión y sostenibilidad de las inversiones dirigidas a niños y niñas en la franja de cero a seis años, especialmente aquellos en condiciones de vulnerabilidad. 
La Organización de Estados Americanos, OEA, también contribuyó a posicionar lo trascendental de las inversiones en primera infancia y ha destinado recursos al diseño y ejecución de programas a favor de este grupo poblacional en sus países miembros. Promulga que esta es una fase decisiva en el ciclo de la vida del ser humano. Destaca con la evidencia de la psicología, biología, nutrición y otros campos de la investigación que los primeros años son críticos para el comportamiento socio emocional y el desarrollo físico e intelectual de los niños. Recientemente su directora Leonore Yafee García, manifestó que "la época más crítica en el desarrollo humano es la primera infancia y un factor clave en el aprendizaje es la calidad de los docentes, por lo que debería surgir un imperativo a los más altos niveles de la política local, nacional e internacional" ${ }^{1}$.

\subsection{Programas e inversiones altamente rentables para la primera infancia}

Uno de los programas pioneros en primera infancia es el HighSchope/Perry Preschool, desarrollado en Míchigan (E.U.), ampliamente documentado a partir de un estudio longitudinal (citado en Santibáñez L; Vegas E, 2009). Este proyecto se centró en brindar medio día de educación preescolar a 123 niños entre tres y cuatro años de edad más una visita semanal a la madre y al niño en su hogar, durante siete meses y medio de cada uno de los dos años de la intervención. El grupo de beneficiarios residía en sectores empobrecidos y las evaluaciones al programa se realizaron hasta que los niños llegaron a los 40 años de edad; esta intervención demostró que los niños alcanzaron un mejor desempeño en las pruebas de lenguajes y cognitivas. De igual modo, los adultos que participaron en el programa lograron ingresos superiores a los que no participaron.

De igual manera, el programa Carolina Abecedarian (citado en Santibáñez L; Vegas E, 2009), demostró resultados positivos en la vida de los niños y niñas. Éste se realizó en Carolina del Norte (E.U.) y brindó un servicio intensivo y controlado a niños desde su nacimiento hasta los 5 años de edad, durante ocho horas diarias de cuidado infantil en un centro de atención, cinco días a la semana. Al ingresar los pequeños a la escuela primaria se encontró que éstos obtuvieron puntajes más altos en las pruebas de logros, repitieron menos grados y recibieron menos educación especial que los niños que no participaron en el programa. Así mismo, al llegar a la edad de 21 años tuvieron puntajes promedios más altos en las pruebas y el doble de posibilidad de permanecer en una escuela o asistir a una universidad que los adultos jóvenes que no se habían beneficiado del programa.

1 Intrazacatecas (2009). OEA impulsa programas primera infancia. En: Página web http://ntrzacatecas.com/noticias/mexi-
co/2009/09/23/impulsaoea-programa-de-educacion-a-primera-infancia/. Fecha de consulta: 23 de octubre de 2011 
En América Latina y el Caribe también se han realizado diversos programas para promover el desarrollo de la primera infancia, entre los cuales, retomando a Emiliana Vegas y Lucrecia Santibáñez en su texto La promesa del desarrollo, se destacan los siguientes: 'Conozca a su hijo', CASH, (Chile); 'Proyectos de atención integral a niños y niñas' menores de seis años de la Sierra Rural, PAIN, (Perú); 'Atención integral a la niñez comunitaria', AIN-C, (Honduras); 'Proyecto nutrición y protección social', AINC-C, para la nutrición y protección (Honduras), Hogares comunitarios y familias en acción (Colombia); Oportunidades (México) y Madres guías (Honduras), entre otras intervenciones con las que se ha logrado contribuir a mejorar el desarrollo temprano de los niños que participaron en los mismos.

En la costa Caribe colombiana son conocidos los aportes de la Universidad del Norte en el diseño e implementación de programas de atención a la primera infancia (Amar, J. Alcalá, M., 2002). En este sentido, es oportuno resaltar algunos: Proyecto de atención integral a la infancia "Costa Atlántica"; Proyecto de desarrollo humano a partir del modelo ecológico de Bronfenbrenner, dirigido al cuidado de los niños, las familias y la comunidad en condiciones de riesgo ambiental, Proyecto alianza para el cuidado de la infancia; Proyecto para la erradicación y la prevención del trabajo infantil en la minería artesanal de Toluviejo (Sucre) y el fortalecimiento de ambientes de cuidado (Amar, J. Madariaga, C., 2008).

Los resultados de los programas referenciados justifican la inversión en primera infancia. Esta inversión se estima en, por lo menos, tres dólares y posiblemente hasta 18 por cada dólar invertido, según el economista y econometrista de la Universidad Erasmus de Rotterdam, Jacques Van Der Gaag, quien retoma la conclusión de expertos del Banco Mundial para afirmar que no puede haber progreso de una nación si no se invierte en el desarrollo temprano del niño.

En la última década, Colombia asumió el reto de poner en la agenda pública la importancia de la inversión a la primera infancia, y ha avanzado en la movilización del tema y en el diseño de políticas públicas. No obstante, los programas a largo plazo y la inversión de recursos adecuados son solo dos de los retos a los que se enfrenta esta nación para el cumplimiento de su compromiso ético con los niños menores de seis años que, al contrario de lo que se piensa, no son su futuro sino su presente, su realidad actual y su oportunidad de borrar la pobreza, la inequidad, la semillas de la violencia y de la injusticia, para ser un mejor pueblo. 


\section{PRIMERA INFANCIA EN COLOMBIA. NORMATIVIDAD, ALGUNOS PROGRAMAS Y BREVE TRAYECTORIA DE LAS POLÍTICAS PÚBLICAS}

En este apartado se registran algunos programas de atención a la primera infancia en Colombia y las principales políticas públicas orientadas a esta población. Siguiendo a Roth (2003) se entienden éstas como una serie de procesos de toma de decisiones y acciones que son promovidas por los gobiernos y por diversos actores de la sociedad civil con el propósito de hacer frente a problemas de la sociedad y definir acciones tanto de promoción como de prevención o atención. Su construcción sigue un ciclo descrito por Salazar (1995), esto es: a) origen, creación o gestación; b) formulación; c) puesta en marcha o implementación; d) evaluación; e) análisis, y f) reformulación o reestructuración.

\subsection{Los primeros escenarios}

La trayectoria de atención a la primera infancia en el país se registra de manera detallada en Colombia por la Primera Infancia². En 1962 el Ministerio de Educación Nacional creó los Jardines Infantiles Nacionales. En 1968 se destaca la creación del Instituto Colombiano de Bienestar Familiar, ICBF, mediante la Ley 75 de 1968. Años más tarde, se llevó a cabo la apertura de los Centros de Atención Integral al Preescolar, CAIP, mediante la Ley 27 de 1974. En 1976, mediante el Decreto No.088 se incluye la educación preescolar como el primer nivel del sistema educativo formal por parte del Ministerio de Educación Nacional. La atención nutricional recibió atención con el Plan Nacional de Alimentación y Nutrición, PAN, inserto en el Plan de Desarrollo "Para Cerrar la Brecha" (1974-1978).

En 1979 se da inicio al Sistema Nacional de Bienestar Familiar (SNBF) por medio de la Ley 7 de 1979. Este sistema estableció las normas para proteger a los niños y las niñas, promover la integración familiar, garantizar los derechos de los niños y de las niñas y ejercer funciones de coordinación de las entidades estatales, relacionadas con los problemas de la familia y del menor.

En la década de los 80 se diseñó el Plan Nacional para la Supervivencia y el Desarrollo Infantil, SUPERVIVIR, orientado a mejorar la salud y la nutrición de la población infantil.

Ministerio de Educación ICBF (2006). Política Pública de Primera Infancia Colombia por la Primera Infancia. En: Página web http://www.cinde.org.co/ PDF/Politica\%20publica\%20 primera\%20infancia\% 20 Colombio\% 20 (vo Colompia 20 (v.\%2011\%20 nov\%2006). pdf. Fecha de consulta: 25 de septiembre de 2011 
En 1986 se implementó el programa más ambicioso del que se tenga noticias en Colombia, que tiene como objetivo atender a la población infantil: el Programa de Hogares Comunitarios de Bienestar, HCB. La atención de los infantes quedó bajo la responsabilidad de las madres comunitarias. En la Ley General de Educación (Ley 115 de 1994) se institucionalizó el Grado Cero que amplió las coberturas de atención, mejoró la calidad y avanzó en la promoción del desarrollo armónico de los niños y niñas.

En la década del 90, se da inicio al Programa Nacional de Acción en Favor de la Infancia, PAFI, el cual se alinea al compromiso de Colombia con las metas previstas en la Cumbre Mundial de la Infancia. Así mismo se crea el Programa de Educación Familiar para el Desarrollo Infantil, PEFADI, que se estableció para favorecer el desarrollo infantil temprano.

Por su parte, el Sistema General de Seguridad Social en Salud, priorizó la atención de madres gestantes y lactantes de la población infantil menor de un año y de las mujeres cabeza de familia (Ley 100 de 1993). El ICBF creó el Programa Fami (Familia, Mujer e Infancia) que además de orientar a las mujeres gestantes y lactantes, suministra complemento nutricional; las responsables de ejecutarlo son mujeres de distintas comunidades y su cobertura es nacional.

\subsection{Escenarios recientes}

Uno de los compromisos de Colombia con la comunidad internacional es poner en práctica el mandato de la Convención de los Derechos del Niño, ratificada mediante Ley 12 de 1991. Ésta tiene carácter vinculante, es decir, que los países firmantes deben prestar especial atención a la garantía de los derechos de los niños y niñas. El Comité Internacional de los Derechos del Niño hace seguimiento al cumplimiento de los compromisos con los derechos de los niños y solicita informes a los países para monitorear avances.

La legislación colombiana, en los últimos años, se ha adaptado a los requerimientos de la Convención de los Derechos del Niño. En el 2006 se pasó del Código del Menor, centrado en la situación irregular de niños y niñas, al Código de Infancia y Adolescencia, expedido mediante Ley 1098 de noviembre de 2006, basado en la protección integral. El artículo 29 de este Código muestra avances en relación con la primera infancia que es definida como la etapa del ciclo vital en la que se establecen las bases para el desarrollo 
cognitivo, emocional y social del ser humano, y que comprende la franja de población de cero a seis años de edad (Ley 1098 de 2006, artículo 29)³.

Es la primera vez que en la legislación colombiana se define de manera explícita la primera infancia y sus derechos, lo que refleja la importancia que este grupo poblacional ha ido cobrando en los últimos años y el fortalecimiento de las políticas públicas para promover su desarrollo y garantizar sus derechos. Importancia que requiere traducirse de manera más contundente en acciones articuladas, suficientemente financiadas y proyectadas a largo plazo con un seguimiento y monitoreo riguroso.

Sin duda, la evidencia más clara del interés de la sociedad y el Estado colombiano en el tema de la primera infancia fue el proceso iniciado en el 2003, con el liderazgo de organizaciones públicas y privadas articuladas en la Alianza por una Política Pública de Infancia y Adolescencia. En 2003, el país se puso a la tarea de apostarle al desarrollo de la primera infancia y poner en relieve la responsabilidad de diversos actores en la garantía de derechos.

Este ejercicio colectivo tuvo como resultado la formulación de la política pública "Colombia por la Primera Infancia", construida en torno a una serie de reflexiones que se hicieron en varios foros internacionales que lograron llamar la atención sobre la primera infancia, la secuencia y los temas específicos de estos foros se recogen en la siguiente tabla:

Tabla 1. Secuencia y temas específicos de los foros por la primera infancia realizados en Colombia

\begin{tabular}{|c|l|l|}
\hline Año & \multicolumn{1}{|c|}{ Foro } & \multicolumn{1}{|c|}{ Propósito central } \\
\hline $\mathbf{2 0 0 3}$ & $\begin{array}{l}\text { I Foro Internacional "Primera Infancia } \\
\text { y Desarrollo: El desafío de la década" }\end{array}$ & $\begin{array}{l}\text { Reposicionar y resignificar las te- } \\
\text { máticas del desarrollo de la primera } \\
\text { infancia. }\end{array}$ \\
\hline $\mathbf{2 0 0 5}$ & $\begin{array}{l}\text { II Foro Internacional: Movilización } \\
\text { por la Primera Infancia" }\end{array}$ & $\begin{array}{l}\text { Indagar y proponer estrategias para } \\
\text { avanzar en la definición de la política } \\
\text { pública }\end{array}$ \\
\hline $\mathbf{2 0 0 7}$ & $\begin{array}{l}\text { III Foro Internacional para la Prime- } \\
\text { ra Infancia. "Acciones para Primera } \\
\text { Infancia" }\end{array}$ & $\begin{array}{l}\text { Intercambiar experiencias exitosas en } \\
\text { la atención integral a los niños desde } \\
\text { la gestación hasta los seis años de edad }\end{array}$ \\
$\begin{array}{l}\text { Infancia. "Sociedad Civil, Estado y y } \\
\text { Primera Infancia" }\end{array}$ & $\begin{array}{l}\text { Consolidar una cultura garante de la } \\
\text { atención y los derechos de la primera } \\
\text { infancia y estrechar los vínculos so- } \\
\text { ciedad civil-estado. }\end{array}$ \\
\hline
\end{tabular}


Otro elemento clave para la formulación de este proceso, fue el Programa de Apoyo a la Formulación de la Política Pública de Primera Infancia, en el cual intervinieron diversos actores nacionales y regionales. El documento que resultó de este ejercicio fue la base para que el gobierno del momento lanzara el CONPES 109 de 2007, que formalizó la política pública para los menores de seis años (Colombia por la Primera Infancia) ${ }^{4}$.

El objetivo de la política "Colombia por la Primera Infancia" se formuló con el objetivo de "promover el desarrollo integral de los niños y niñas desde la gestación hasta los 6 años de edad; respondiendo a sus necesidades y características específicas, y contribuyendo así al logro de la equidad e inclusión social en Colombia".

Algunos objetivos específicos de esta política fueron ${ }^{5}$ :

Fortalecer y ampliar la atención en educación inicial con enfoque integral en los denominados entornos institucional, familiar y comunitario, asegurando la sostenibilidad de los recursos para su funcionamiento

Darle un lugar al tema de primera infancia en el país, sensibilizando diversos actores y generando un proceso de movilización. Trabajar en el diseño de mecanismos para la formulación, implementación, seguimiento y evaluación de políticas públicas para la franja poblacional de cero a seis.

A lo anterior se agrega que, en coherencia con el corto plazo de las políticas públicas en Colombia, el actual gobierno ha lanzado una nueva estrategia como política de atención a la primera infancia, sobre la que se hablará en el apartado 2.3.

${ }^{4}$ Ministerio de Educación (2011). Primera infancia. En: Página web http:// www.mineducacion.gov. $\mathrm{co} /$ primerainfancia/ 1739 articles 177828 archivo pdf conpes 109. $\mathrm{p} d f$. Fechā de consulta: 22 de octubre de 2011

${ }^{5}$ OEI (2006). Colombia por la Primera Infancia. En: Página Web http://www. oei.es/quipu/colombia politica_primer_infancia pdf. Fecha de consulta: 26 de septiembre de 2011

En el gobierno de Álvaro Uribe Vélez, el Ministerio de Educación Nacional lideró la creación de la Política Educativa para la Primera Infancia, que fue presentada en el 2009. Éste proyecto ha recibido críticas por los bajos niveles de participación de actores relevantes en su formulación. Su objetivo es garantizar una mejor calidad de atención integral (salud, nutrición y educación inicial) a los niños y niñas entre los cero y cinco años, especialmente los pertenecientes a los niveles uno y dos del Sistema de Identificación y Clasificación de Potenciales Beneficiarios, SISBEN. Se aspira a que la primera infancia reciba atención integral mediante programas que involucren a la familia, la comunidad y las instituciones especializadas.

Palobra No. 12. Agosto de 2010 - Julio de 2011 
La Política Educativa de Primera Infancia, que inicialmente se propuso atender 400.000 niños y niñas, se enmarca en la perspectiva de derechos y recoge avances de países como Cuba en el Programa educa tu hijo. Esta política cuenta con las siguientes modalidades de atención: entorno familiar, comunitario e institucional. Las modalidades de atención funcionan con recursos asignados para tal fin a través del CONPES 123 de 2009. Actualmente los recursos son administrados y acompañados técnicamente por el Fondo Financiero de Proyectos de Desarrollo, FONADE, que suscribió un contrato por tres mil doscientos millones de pesos (3.200.000.000,00) con el Ministerio de Educación Nacional.

La alianza entre el Ministerio de Educación y el Instituto Colombiano de Bienestar Familiar, esperada por el país, fue producto de esta política; tradicionalmente, estas dos entidades han realizado trabajos individuales, sin articulación. La atención a los niños es brindada por operadores locales que suscriben convenios para la prestación del servicio.

También hay que destacar la Ley 1295 de 2009, que reglamenta la atención integral de los niños menores de seis años, pertenecientes a los niveles uno, dos y tres del SISBEN. En su artículo primero se lee que su objetivo es mejorar la calidad de vida de las madres gestantes, niñas y niños menores de seis años, clasificados en los estratos anteriormente mencionados, y que obliga al Estado colombiano a garantizarles sus derechos a la alimentación, la nutrición adecuada, la educación inicial y la atención integral en salud (Ley 1295 de 2009, artículo 1).

\subsection{Momento actual}

Actualmente, el país tiene en marcha una nueva política de atención integral a la primera infancia, inserta en el Plan de Desarrollo 2010- 2014. Esta ha sido denominada "De cero a siempre" y se le definió como un "conjunto de acciones planificadas, de carácter nacional y territorial, dirigidas a promover y garantizar el mejoramiento infantil temprano a través de un trabajo unificado e intersectorial, que desde una perspectiva de derechos, articula y promueve los planes, programas y acciones que adelanta el país en favor de la atención integral a la primera infancia" ${ }^{6}$. Los objetivos específicos de esta estrategia son los siguientes: 


\section{Objetivos Estrategia De cero a siempre}

Garantizar el cumplimiento de los derechos de las niñas y los niños en primera infancia.

Definir una política pública de largo plazo que oriente al país en materia de sostenibilidad técnica y financiera, universalización de la atención y fortalecimiento de los territorios.

Garantizar la pertinencia y calidad en la atención integral a la primera infancia, articulando acciones desde antes de la concepción, hasta la transición hacia la educación formal.

Sensibilizar y movilizar a toda la sociedad colombiana con el propósito de transformar las concepciones y formas de relación con los niños y las niñas más pequeños. Hacer visible y fortalecer a la familia como actor fundamental en el desarrollo infantil temprano.

Fuente: Estrategia de cero a siempre (presentación), Octubre de 2011. Bogotá

Como metas de la estrategia se pretende brindar atención integral con calidad a un millón doscientos mil (1.200.000) niños y niñas y sus familias con prioridad en la población del Programa Unidos de la Acción Social, construcción de más de 400 Centros de Desarrollo Infantil, formación a más de 100.000 agentes educativos en diferentes aspectos de la estrategia, transición de más de quinientos mil (500.000) niños y niñas de Hogares Comunitarios a una nueva forma de atención.

La Estrategia "de cero a siempre" expresa la voluntad política del actual gobierno, encaminada, según el Departamento Administrativo Nacional de Estadística, DANE (Censo 2005, proyecciones para el 2010), a beneficiar a 2.875.634 niños que conforman el 56\% de los más de cinco millones que habitan el país. La inversión de la misma sería de 5.4 billones de pesos para la construcción de centros de desarrollo infantil e itinerante en los cuatro años de la administración del gobierno del presidente Juan Manuel Santos. Expertos como Jorge Oroza, economista vinculado a la oficina de Save the Children para la región de América Latina, reconoce el avance en Colombia pero asegura que aún queda camino por recorrer.

En cualquier caso, son grandes las expectativas que hay sobre el desarrollo de la Estrategia de Cero a Siempre y en los avances que puede reportar en materia de atención integral a la primera infancia no solo en este gobierno sino a largo plazo.

\section{CONCLUSIONES Y RECOMENDACIONES}

El abordaje de un tema vital como la primera infancia permite concluir que los esfuerzos de Colombia constituyen un aporte valioso, pero todavía limitado 
para seguir avanzando en la consolidación de una cultura que permita ubicar en lugar justo la atención integral de los niños y niñas entre cero y seis años. La movilización del tema, el diseño de políticas serias, la inclusión en la agenda pública y la comprensión de la importancia de invertir en el desarrollo infantil temprano puede considerarse como avances en apertura de nuevas rutas que debe navegar el país en la búsqueda de la construcción de una mejor sociedad.

El compromiso ineludible de la Nación es generar políticas públicas de largo plazo destinadas a favorecer el desarrollo de la infancia con inversiones, recursos y procesos de monitoreo que permitan alcanzar un gran potencial humano en beneficio del Estado y la sociedad.

En la actualidad, si bien es cierto que se ha contado con el apoyo de organismos internacionales para impulsar el fortalecimiento de la población infantil vulnerable, se puede concluir que la política pública de primera infancia del presente gobierno "de cero a siempre" no será suficiente para atender a todos los niños y niñas por fuera del sistema, actualmente calculados en más de dos millones. En todo caso, la nueva estrategia avanza en un momento de implementación, es necesario, esperar el desarrollo de las fases de evaluación, análisis y reformulación para asumir unas posturas definitivas frente a sus indicadores.

Finalmente, se recomienda generar iniciativas que comprometan al parlamento Colombiano, para que la comisión de presupuesto asigne de manera prioritaria la mejor disponibilidad de recursos a la inversión de la primera infancia. Iniciativas de esta envergadura tienen como condición imprescindible la sustentación de los argumentos científicos que obligan a invertir en los niños y niñas.

De igual forma, es oportuno retomar las contundentes experiencias de la Universidad del Norte, la Universidad de Manizales y el Centro Internacional de Educación y Desarrollo Humano, CINDE, con su programa de Doctorado en Ciencias Sociales, Niñez y Juventud, la Universidad Nacional y la Universidad del Valle, para avanzar en la formación de expertos en el tema, a nivel de educación postgraduada, para que estos, a su vez potencien, de mejor manera, el talento humano en las distintas regiones del país, donde se requieren nuevos y mejores aportes a la franja importante que incide en los niños: madres comunitarias, promotores, animadores, voluntarios, monitores, docentes y cuidadores.

Palubra No. 12. Agosto de 2010 - Julio de 2011 


\section{BIBLIOGRAFÍA}

Amar, J. Alcalá, M. (2002). Políticas sociales y modelos de atención integral a la primera infancia. En el marco del Segundo Foro Internacional: Movilización por la primera infancia. Bogotá: Ediciones Uninorte.

Amar, J. Madariaga, C. (2008). Proyectos sociales y cuidados a la infancia. Ediciones Uninorte. Barranquilla.

Banco Mundial. (2002). Directorio de proyectos de desarrollo infantil temprano en América Latina y el Caribe: Autor.

Convención sobre los Derechos de los Niños. Bogotá: Unicef.

Compendio de leyes Colombianas (s.f.p.): Ley 75 de 1968; Ley 27 de 1974; Ley 7 de 1979; Ley 12 de 1991; Ley 100 de 1993; Ley 115 de Febrero 8 de 1994.; Ley 1295 de 2009; Ley 1098 de 2006.

Departamento Nacional de Planeación, Instituto Colombiano de Bienestar Familiar, Ministerio de Educación Nacional y Ministerio de Protección Social. (2007). Documento CONPES Social 109. Política pública nacional de primera infancia "Colombia por la primera infancia". Bogotá: Autores.

Departamento Nacional de Planeación, Instituto Colombiano de Bienestar Familiar, Ministerio de Educación Nacional, Ministerio de Protección Social. (2009). Documento CONPES Social 123. Concejo Nacional de Política Económica y Social. Bogotá: Autores.

DANE (2005), Censo 2005.

Estrategia De cero a siempre (presentación), Octubre de 2011. Bogotá.

Encuesta Nacional de Demografía y Salud. (2010). Bogotá.

FONDO PARA LA ACCIÓN AMBIENTAL Y LA NIÑEZ, ICBF (2006), «Movilización por la Primera Infancia», II Foro. Memorias, Edición: FPAA - ICBF.

INSTITUTO COLOMBIANO DE BIENESTAR FAMIIIAR; ALCALDÍA MAYOR DE BOGOTÁ; SAVE THE CHILDREN; UNICEF Y CINDE (2003), Primera infancia y desarrollo. El desafío de la década, Bogotá: Cargraphics.

III Foro Internacional para la Primera Infancia. "Acciones para Primera Infancia". (2007).

IV Foro Internacional de Primera Infancia. "Sociedad Civil, Estado y Primera Infancia". (2009).

Heckman, J. (2010). Reflexiones sobre importancia de invertir en la primera infancia. www. heckmanequation.org

Ministerio de Educación Nacional. (2010). Programa de atención integral a la primera infancia. PAIPI. Bogotá D, C.: Autor.

MUSTARD, J. F. (2003). Desarrollo infantil inicial: Salud, aprendizaje y comportamiento. En ICBF; Alcaldía Mayor de Bogotá, Save the Children UK, UNICEF y CINDE. (Ed.). Primera Infancia y Desarrollo. El desafío de la Década. Bogotá: Cargrapships. 
PROFAMILIA (2005), Encuesta Nacional de Demografía y Salud. 2010, Bogotá: Profamilia.

ROTH, D. (2003). Políticas públicas: formulación, implementación y evaluación, Bogotá, ediciones Aurora.

SALAZAR, C. Las políticas públicas. Medellín, Colección jurídicas, Profesores No 19,1995.

SANTIBAÑEZ, L. y Vegas, E. (2009). La promesa del desarrollo en la primera infancia en América Latina y el Caribe. Bogotá: Mayol ediciones.

SEN, A. (1999). Romper el ciclo de la pobreza: Invertir en la infancia", París: (s.n.).

VAN DER Gaar, J. (2006). La inversión en la primera infancia como política pública. En FPPA - ICBF. (Ed.), II Foro Internacional: Movilización por la Primera Infancia. Bogotá: FPPA - ICBF.

YOUNG, M. (2003, Febrero). Aprendizaje temprano, ganancia futura. Human development network World Bank. Ponencia presentada en el foro primera infancia y desarrollo: el desafío de la década, Bogotá.

\section{CIBERGRAFÍA}

ICBF (2011). Instituto Colombiano de Bienestar Familiar. En: Página web www.icbf.gov.co. Fecha de consulta: 20 de octubre de 2011

Ministerio de Educación (2011). Primera infancia. En: Página web http://www.mineducacion. gov.co/primerainfancia/1739/articles177828_archivo_pdf_conpes109.pdf. Fecha de consulta: 22 de octubre de 2011

Intrazacatecas (2009). OEA impulsa programas primera infancia. En: Página web http:// ntrzacatecas.com/noticias/mexico/2009/09/23/impulsa-oea-programa-de-educacion-a-primerainfancia/. Fecha de consulta: 23 de octubre de 2011

Ministerio de Educación, ICBF (2006). Política Pública de Primera Infancia Colombia por la Primera Infancia. En: Página web http://www.cinde.org.co/PDF/Politica\%20publica\%20primera\%20 infancia\%20Colombia\%20(v.\%2011\%20nov\%2006).pdf. Fecha de consulta: 25 de septiembre de 2011

OEI (2006). Colombia por la Primera Infancia. En: Página Web http://www.oei.es/quipu/colombia/ politica_primer_infancia.pdf. Fecha de consulta: 26 de septiembre de 2011

Palobra No. 12. Agosto de 2010 - Julio de 2011 